\title{
On global continua of solutions bifurcating from zero and infinity of some nonlinear fourth order eigenvalue problems
}

\author{
Natavan A. Mustafayeva *
}

Received: 28.08.2019 / Revised: 16.02.2020 / Accepted: 03.05.2020

Abstract. In this paper we consider the nonlinear eigenvalue problems for ordinary differential equations of fourth order. The previously obtained results on global bifurcation of solutions from zero and infinity of these problems in wider classes of functions with fixed oscillation count are established.

Keywords. nonlinear eigenvalue problem, eigenfunction, bifurcation from zero, bifurcation from infinity, global continuum of solutions

Mathematics Subject Classification (2010): 34B05, 34B15, 34C23, 47H11, 47J10, 47J15

\section{Introduction}

We consider the following nonlinear boundary value problem

$$
\begin{gathered}
\left.\ell(y) \equiv\left(p(x) y^{\prime \prime}\right)^{\prime \prime}-\left(q(x) y^{\prime}\right)^{\prime}+r(x) y=\lambda \tau(x) y+g\left(x, y, y^{\prime}, y^{\prime \prime}, y^{\prime \prime \prime}, \lambda\right)\right) y, x \in(0, l), \\
y^{\prime}(0) \cos \alpha-\left(p y^{\prime \prime}\right)(0) \sin \alpha=0, \quad y(0) \cos \beta+T y(0) \sin \beta=0, \\
y^{\prime}(l) \cos \gamma+\left(p y^{\prime \prime}\right)(l) \sin \gamma=0, \quad y(l) \cos \delta-T y(l) \sin \delta=0
\end{gathered}
$$

where $\lambda \in \mathbb{R}$ is a spectral parameter, $T y \equiv\left(p y^{\prime \prime}\right)^{\prime}-q y^{\prime}$. Regarding the coefficients $p, q, r, \tau$ of the equation (1.1) and the coefficients $\alpha, \beta, \gamma, \delta$ of boundary conditions (1.2) we assume that $p \in C^{2}([0, l] ;(0,+\infty)), q \in C^{1}([0, l] ;(0,+\infty)), r \in C^{0}([0, l] ; \mathbb{R})$, $\tau \in C^{0}([0, l] ;(0,+\infty))$ and $\alpha, \beta, \gamma, \delta$ are real constants such that $0 \leq \alpha, \beta, \gamma, \delta \leq \frac{\pi}{2}$. Moreover, $g \in C^{0}\left([0, l] \times \mathbb{R}^{5} ; \mathbb{R}\right)$ and satisfies one or both of the following conditions:

$$
\begin{gathered}
g(x, y, s, v, w, \lambda)=o(|y|+|s|+|v|+|w|) \text { as }|y|+|s|+|v|+|w| \rightarrow 0 ; \\
g(x, y, s, v, w, \lambda)=o(|y|+|s|+|v|+|w|) \text { as }|y|+|s|+|v|+|w| \rightarrow \infty,
\end{gathered}
$$

uniformly in $x \in[0, l]$ and $\lambda \in \Lambda$ for each finite interval $\Lambda \subset \mathbb{R}$.

From the middle of the last century to the present, the local and global theory of bifurcation of nonlinear eigenvalue problems has been intensively developed and very substantial results have been obtained in these directions (see, for example, [1-3], [4-25]). Nonlinear

\footnotetext{
* Corresponding author
}

N.A. Mustafayeva

Ganja State University, Ganja, AZ2000, Azerbaijan

E-mail: natavan1984@gmail.com 
eigenvalue problems for ordinary differential equations arise in mechanics, physics, chemistry, and also in various fields of natural science (see, for example, [12-14]). Therefore, the study of these types of problems is naturally of great interest.

Local and global bifurcation from zero and infinity of solutions of non-linear eigenvalue problems for ordinary differential equations of the second and fourth orders, a system of differential equations of the first order and partial differential equations of elliptic type are investigated in detail in [1-3, 5-7, 9-11, 19-25].

Note that if condition (1.3) holds, then we consider bifurcation from zero, if condition (1.4) holds, then we consider bifurcation from infinity, and if both conditions (1.3) and (1.4) are satisfied simultaneously, then we consider bifurcation from zero and infinity at the same time.

Problem (1.1), (1.2) in the case when nonlinear term $f=g y$ has a more general form was considered in $[2,3,9]$. Since all the eigenvalues $\lambda_{k}, k=1,2, \ldots$, of the linear problem

$$
\begin{gathered}
\ell(y)(x)=\lambda \tau(x) y(x), x \in(0, l), \\
y^{\prime}(0) \cos \alpha-\left(p y^{\prime \prime}\right)(0) \sin \alpha=0, \quad y(0) \cos \beta+T y(0) \sin \beta=0, \\
y^{\prime}(l) \cos \gamma+\left(p y^{\prime \prime}\right)(l) \sin \gamma=0, \quad y(l) \cos \delta-T y(l) \sin \delta=0,
\end{gathered}
$$

are real and simple (see $[3,4])$ it follows that $\left(\lambda_{k}, 0\right)$ is a bifurcation point respect to the line $\mathcal{R}=\left\{(\lambda, 0) \in \mathbb{R} \times C^{3}([0,1] ; \mathbb{R}): \lambda \in \mathbb{R}\right\}$ of trivial solutions of problem (1.1), (1.2) if satisfies the condition (1.3) and $\left(\lambda_{k}, \infty\right)$ is a asymptotic bifurcation point (i.e. bifurcation point respect to the set $\mathfrak{R}=\left\{(\lambda, \infty) \in \mathbb{R} \times C^{3}([0,1] ; \mathbb{R}): \lambda \in \mathbb{R}\right\}$ ) of problem (1.1), (1.2) if satisfies the condition (1.4). In [3], the author constructed a class $S_{k}^{\nu}, k \in \mathbb{N}, \nu \in\{+,-\}$, of functions $\{y\}$ of Banach space

$$
E=C^{3}([0,1] ; \mathbb{R}) \cap B . C .
$$

with usual norm

$$
\|y\|_{3}=\sum_{s=0}^{3}\left\|y^{(s)}\right\|_{\infty},\|y\|_{\infty}=\max _{x \in[0, l]}|y(x)|,
$$

that the functions $\{\nu y\}$ are positive near $x=0$ and have oscillatory properties of the $k$ th eigenfunction of problem (1.5) and their derivatives, where by B.C. we denote the set of functions satisfying the boundary conditions (1.2). In [3], in particular, it was shown that for each $k \in \mathbb{N}$ and each $\nu \in\{+,-\}$ there exists a continuum $C_{k}^{\nu}$ of solutions of problem (1.1), (1.2) which contains $\left(\lambda_{k}, 0\right)$, lies in $\left(\mathbb{R} \times S_{k}^{\nu}\right) \cup\left\{\left(\lambda_{k}, 0\right)\right\}$ and is unbounded in $\mathbb{R} \times E$ (see [3, Theorem 1.1]). If the condition (1.4) is satisfies, then in [9] it was proved that for each $k \in \mathbb{N}$ and each $\nu \in\{+,-\}$ there exists a connected component $D_{k}^{\nu}$ of solutions of problem (1.1), (1.2) which meets $\left(\lambda_{k}, \infty\right)$ and has the following properties: (i) there exists a neighborhood $Q_{k}$ of $\left(\lambda_{k}, \infty\right)$ in $\mathbb{R} \times E$ such that $Q_{k} \cap\left(D_{k}^{\nu} \backslash\left(\lambda_{k}, \infty\right)\right) \subset\left(\mathbb{R} \times S_{k}^{\nu}\right)$; (ii) either $D_{k}^{\nu}$ meets $D_{k^{\prime}}^{\nu^{\prime}}$ through $\mathbb{R} \times S_{k^{\prime}}^{\nu^{\prime}}$ for some $\left(k^{\prime}, \nu^{\prime}\right) \neq(k, \nu)$, or $D_{k}^{\nu}$ meets $(\lambda, 0)$ for some $\lambda \in \mathbb{R}$, or $P_{\mathbb{R}}\left(D_{k}^{\nu}\right)$ is unbounded in $\mathbb{R}$ (see [9, Theorem 3.1]). Moreover, there it was shown that if both conditions (1.3) and (1.4) are satisfied, then for each $k \in \mathbb{N}$ and each $\nu \in\{+,-\}$ we have $D_{k}^{\nu} \subset \mathbb{R} \times S_{k}^{\nu}$ and alternative (i) cannot hold. Furthermore, if $C_{k}^{\nu}$ $\left(D_{k}^{\nu}\right)$ meets $(\lambda, \infty)(\lambda, 0)$ for some $\lambda \in \mathbb{R}$, then $\lambda=\lambda_{k}$ (see [9, Theorem 4.1]).

The purpose of this paper is to establish global results on bifurcation of solutions from zero and infinity for the problem (1.1), (1.2) in classes $\mathcal{S}_{k}^{\nu}, k \in \mathbb{N}, \nu \in\{+,-\}$, of functions $y \in E$ that satisfy the following conditions:

(i) the function $y$ has exactly $k-1$ simple zeros in $(0,1)$,

(ii) $\nu y$ is positive near $x=0$,

(iii) $\sum_{s=0}^{3}\left|y^{(s)}(x)\right|^{2}>0$ for $x \in[0, l]$. 
2 Global bifurcation from zero of solutions of the boundary value problem (1.1), (1.2)

In this section, we suppose that the condition (1.3) holds.

Without loss of generality we can assume that $\lambda=0$ is not eigenvalue of problem (1.5) (see [21, 22]). Then nonlinear eigenvalue problem (1.1)-(1.2) is reduces to the following nonlinear integro-differential equation

$$
\begin{gathered}
y(x)=\lambda \int_{0}^{l} H(x, t) \tau(t) y(t) y(t) d t \\
+\int_{0}^{l} H(x, t) g\left(t, y(t), y^{\prime}(t), y^{\prime \prime}(t), y^{\prime \prime \prime}(t), \lambda\right) y(t) d t
\end{gathered}
$$

where $H(x, t),(x, t) \in[0, l ; 0, l]$, is the Green's function for the operator corresponding to the differential expression $\ell(y)$ and the boundary conditions (1.2).

We define the operators $L: E \rightarrow E$ and $G: \mathbb{R} \times E \rightarrow E$ as follows:

$$
\begin{gathered}
L y(x)=\lambda \int_{0}^{l} H(x, t) \tau(t) y(t) d t, \\
G(\lambda, y(x))=\int_{0}^{l} H(x, t) g\left(t, y(t), y^{\prime}(t), y^{\prime \prime}(t), y^{\prime \prime \prime}(t), \lambda\right) y(t) d t .
\end{gathered}
$$

It follows from [3, $\S 3.3]$ that $L: E \rightarrow E$ is a linear completely continuous operator and $G: \mathbb{R} \times E \rightarrow E$ is a nonlinear completely continuous operator. Moreover, by virtue of (1.3) the following condition holds:

$$
G(\lambda, y)=o\left(\|y\|_{3}\right) \text { as }\|y\|_{3} \rightarrow 0,
$$

uniformly in $\lambda \in \Lambda$.

In view of (2.1)-(2.3) the problem (1.1), (1.2) can be rewritten in the following equivalent operator equation:

$$
y=\lambda L y+G(\lambda, y) .
$$

Should be noted that the sets $S_{k}^{+}, S_{k}^{-}$and $S_{k}=S_{k}^{+} \cup S_{k}^{-}$are open subsets of $E$, and $S_{k}^{\nu} \cap S_{k}^{\prime \nu^{\prime}}=\emptyset$ for each $\left(k^{\prime}, \nu^{\prime}\right) \neq(k, \nu)$. Moreover, if $y \in \partial S_{k}$, then either there exists $\zeta \in(0, l)$ such that $y(\zeta)=y^{\prime}(\zeta)=0$ or there exists $\varsigma \in[0, l]$ such that $y(\varsigma)=y^{\prime}(\varsigma)=$ $y^{\prime \prime}(\varsigma)=y^{\prime \prime \prime}(\varsigma)=0$.

Lemma 2.1 Let $(\mu, u) \in \mathbb{R} \times E$ be a solution of problem (1.1), (1.2) such that $u \in \partial S_{k}$ for some $k \in \mathbb{N}$. Then $u \equiv 0$.

Proof. It is obvious that if $(\mu, u) \in \mathbb{R} \times E$ is a solution of the nonlinear eigenvalue problem (1.1), (1.2), then $(\mu, u)$ solves the following linear eigenvalue problem

$$
\begin{aligned}
& \ell y(x)+\varphi(x) y(x)=\lambda \tau(x) y(x), x \in(0, l), \\
& y \in \text { B.C. }
\end{aligned}
$$

where

$$
\varphi(x)=-g\left(x, u(x), u^{\prime}(x), u^{\prime \prime}(x), u^{\prime \prime \prime}(x), \mu\right), x \in[0, l] .
$$

Since $g \in C^{0}\left([0, l] \times \mathbb{R}^{5} ; \mathbb{R}\right)$ it follows that $\varphi \in C^{0}([0, l] ; \mathbb{R})$. Then by virtue of $[3$, Theorem 1.2] we have

$$
u \in \bigcup_{m=1}^{\infty} S_{m}
$$

which implies that

$$
\partial S_{k} \cap \bigcup_{m=1}^{\infty} S_{m} \neq \emptyset .
$$


But it is obvious that for each $k \in \mathbb{N}$ the relation

$$
\partial S_{k} \cap \bigcup_{m=1}^{\infty} S_{m}=\emptyset
$$

holds. This contradiction completes the proof of Lemma 2.1.

Remark 2.1 If $(\mu, u) \in \mathbb{R} \times E$ be a nontrival solution of problem (1.1), (1.2), then

$$
u \in \bigcup_{k=1}^{\infty} S_{k} .
$$

Theorem 2.1 For each $k \in \mathbb{N}$ and each $\nu \in\{+,-\}$ there exists an unbounded continuum $\mathcal{C}_{k}^{\nu}$ of solutions of problem (1.1), (1.2) such that

$$
\left(\lambda_{k}, 0\right) \in \mathcal{C}_{k}^{\nu} \subset\left(\mathbb{R} \times S_{k}^{\nu}\right) \cup\left\{\left(\lambda_{k}, 0\right)\right\} .
$$

The proof of this theorem is similar to that of [3, Theorem 1.1] with the use of Lemma 2.1 and Remark 2.1.

Theorem 2.2 Let the function $g$ additionally satisfy the following condition:

$$
|g(x, y, s, v, w, \lambda)| \leq M,(x, y, s, v, w, \lambda) \in[0, l] \times \mathbb{R}^{5} .
$$

Then

$$
\mathcal{C}_{k}^{\nu} \subset\left(I_{k} \times S_{k}^{\nu}\right) \cup\left\{\left(\lambda_{k}, 0\right)\right\}
$$

where

$$
I_{k}=\left[\lambda_{k}-\frac{M}{\tau_{0}}, \lambda_{k}+\frac{M}{\tau_{0}}\right], \tau_{0}=\min _{x \in[0, l]} \tau(x) .
$$

Proof. If $(\lambda, y) \in \mathbb{R} \times E$ is a solution of the nonlinear eigenvalue problem (1.1), (1.2) such that $y \in S_{k}^{\nu}$, then it follows from the above that $(\lambda, y)$ is a solution of the linear spectral problem (2.6) with

$$
|\varphi(x)| \leq M \text { for } x \in[0, l] .
$$

Hence it follows from [3, Lemma 4.1] that $\lambda \in I_{k}$. The proof of this theorem is complete.

\section{Global bifurcation from infinity of nontrivial solutions of nonlinear eigenvalue problem (1.1)-(1.2)}

In this section, we assume that the condition (1.4) is satisfied.

Recall that the nonlinear eigenvalue problem (1.1), (1.2) is equivalent to operator equation (2.5). Obviously, in this case the operator $G: \mathbb{R} \times E \rightarrow E$ is continuous. Using (1.4) and by following the arguments in Theorem 2.4 of [22], we can prove that

$$
G(\lambda, y)=o\left(\|y\|_{3}\right) \text { as }\|y\|_{3} \rightarrow \infty,
$$

uniformly in $\lambda \in \Lambda$. Moreover, the operator $\mathcal{G}: \mathbb{R} \times E \rightarrow E$ given by

$$
\mathcal{G}(\lambda, u) \equiv\|y\|_{3}^{2} G\left(\lambda, \frac{y}{\|y\|_{3}^{2}}\right)
$$

is compact. 
Let $D$ denote of the set of solutions of problem (1.1), (1.2). If $(\lambda, y) \in D$ with $y \not \equiv 0$, setting $v=\frac{y}{\|y\|_{3}^{2}}$ and dividing (2.5) by $\|y\|_{3}^{2}$ yields

$$
v=\lambda L v+\mathcal{G}(\lambda, v) .
$$

Setting $\mathcal{G}(\lambda, 0)=0$, we extend $\mathcal{G}$ to $v=0$. Hence the operator $\mathcal{G}$ is continuous in $\mathbb{R} \times E$. Moreover, since $G$ is satisfied (3.1) uniformly in $\lambda \in \Lambda$ for any bounded interval $\Lambda \subset \mathbb{R}$ it follows that

$$
\mathcal{G}(\lambda, y)=o\left(\|y\|_{3}\right) \text { as }\|y\|_{3} \rightarrow 0
$$

uniformly in $\lambda \in \Lambda$.

Theorem 3.1 For each $k \in \mathbb{N}$ and each $\nu \in\{+,-\}$ there exists a connected component $D_{k}^{\nu}$ of $D$ such that $\left(\lambda_{k}, \infty\right) \in D_{k}^{\nu},\left(D_{k}^{\nu} \backslash\left(\lambda_{k}, \infty\right)\right) \subset S_{k}^{\nu}$ and either

(i) $D_{k}^{\nu}$ meets $(\lambda, 0)$ for some $\lambda \in \mathbb{R}$, or

(ii) $P_{\mathbb{R}}\left(D_{k}^{\nu}\right)$ is unbounded in $\mathbb{R}$.

Proof. The proof of this theorem is similar to that of [9, Theorem 3.1] with the use of Lemma 2.1, Remark 2.1, Theorem 2.1 and the above arguments.

Taking into account the proof of Theorem 2.2 from Theorem 3.1, we obtain the following result.

Theorem 3.2 If the function $g$ additionally satisfy the condition (2.7), then

$$
\left(D_{k}^{\nu} \backslash\left(\lambda_{k}, \infty\right)\right) \subset I_{k} \times S_{k}^{\nu}
$$

and the union $D_{k}=D_{k}^{+} \cup D_{k}^{-}$does not satisfy statement (ii) of Theorem 3.1.

\section{Global bifurcation from zero and infinity of nontrivial solutions of problem (1.1)-(1.2)}

In this section we assume that the conditions (1.3) and (1.4) are satisfied simultaneously. Then the statements of Theorems 2.1 and 3.1 are valid at the same time. It should be noted that the question was posed in Section 4 of [9]: do the continua $C_{k}$ and $D_{k}$ intersect? The answer to this question is also given there: constructed the example (see [9, Example 4.2]) which show that, both cases are possible.

Theorem 4.1 Let the function $g$ additionally satisfy the condition (2.7). Then for each $k \in$ $\mathbb{N}$ and each $\nu \in\{+,-\}$ the relations hold:

$$
C_{k}^{\nu} \equiv D_{k}^{\nu}
$$

The proof of this theorem directly follows from [14, Ch. 4, Theorems 2.1, 3.1], Theorems 2.2, 3.2, [3, Theorem 5.1] and [9, Theorem 4.1].

\section{References}

1. Aliyev, Z.S.: Global bifurcation of solutions of certain nonlinear Sturm-Liouville problems, Vestnik Bakin. Univ. Ser. Fiz. Mat. Nauk, 2, 115-120 (2001) (in Russian).

2. Aliyev, Z.S.: Some global results for nonlinear fourth order eigenvalue problems, Cent. Eur. J. Math. 12 (12), 1811-1828 (2014).

3. Aliyev, Z.S.: On the global bifurcation of solutions of some nonlinear eigenvalue problems for ordinary differential equations of fourth order, Sb. Math. 207 (12), 1625-1649 (2016). 
4. Aliev, Z.S., Agaev E.A.: Structure of the root subspaces and oscillation properties of the eigenfunctions of completely regular systems, Proc. Inst. Math. Mech. Natl. Acad. Sci. Azerb. 40 (1), 36-43 (2014).

5. Aliyev Z.S., Hasanova, Sh.M.: Global bifurcation of positive solutions of semi-linear elliptic partial differential equations with indefinite weight, Zeitsch. Anal. Ihre Anwen. 38 (1), 1-15 (2019).

6. Aliyev, Z.S, Huseynova R.A.: Bifurcation in nonlinearizable eigenvalue problems for ordinary differential equations of fourth orderwith indefinite weight, Electron. J. Qual. Theory Differ. Equ. 2017 (92), 1-12 (2017).

7. Aliyev, Z.S. Mamedova, G.M.: Some global results for nonlinear Sturm-Liouville problems with spectral parameter in the boundary condition, Ann. Polon. Math. 115 (1), 75-87 (2015).

8. Aliyev, Z.S., Mustafayeva, N.A.: Bifurcation from infinity for some nonlinear eigenvalue problems which are not linearizable, Trans. Natl. Acad. Sci. Azerb. Ser. Phys.Tech. Math. Sci. Is. Math. 35 (4), 13-18 (2015).

9. Aliyev, Z.S., Mustafayeva, N.A.: Bifurcation of solutions from infinity for certain nonlinear eigenvalue problems of fourth-order ordinary differential equations, Electron. J. Differ. Equ. 2018 (98), 1-19 (2018).

10. Aliev, Z.S., Rzayeva, H.Sh.: Global bifurcation for nonlinear Dirac problems, Electron. J. Qual. Theory Differ. Equ. 2016 (46), 1-14 (2016).

11. Berestycki, H.: On some nonlinear Sturm-Liouville problems, J. Differential Equations, 26 (3), 375-390 (1977).

12. Dickey, R.W.: Bifurcation problems in nonlinear elasticity, Res. Notes Math., V. 3, Pitman Publishing, LondonSan Francisco, California-Melbourne, (1976).

13. Keller, J.B., Antman S. (eds.): Bifurcation theory and nonlinear eigenvalue problems, Mathematics Lecture Note Series, W. A. Benjamin, Inc. New YorkAmsterdam, (1969).

14. Krasnoselskii M.A.: Topological methods in the theory of nonlinear integral equations, Macmillan, New York, 1965.

15. Lazer A.C., McKenna P.J.: Global bifurcation and a theorem of Tarantello, J. Math. Anal. Appl. 181 (3), 648-655, (1994).

16. Lopez-Gomez, J.: Spectral theory and nonlinear functional analysis, Chapman and Hall/CRC Res. Notes Math., V. 426, Chapman and Hall/CRC, Boca Raton, FL (2001).

17. Makhmudov, A.P., Aliev Z.S.: Global bifurcation of solutions of certain nonlinearizable eigenvalue problems, Diff. Equat. 25 (1), 71-76 (1989).

18. Makhmudov, A.P., Aliyev, Z.S.: Nondifferentiable perturbations of spectral problems for a pair of selfadjoint operators and global bifurcation, Soviet Math. 34 (1) 51-60 (1990).

19. Przybycin J.: Bifurcation from infinity for the special class of nonlinear differential equations, J. Differential Equations, 65 (2), 235-239 (1986).

20. Przybycin J.: Some applications of bifurcation theory to ordinary differential equations of the fourth order, Ann. Polon. Math. 53 (2), 153-160 (1991).

21. Rabinowitz P.H.: Some global results for nonlinear eigenvalue problems, J. Funct. Anal. 7 (3), 487-513 (1971).

22. Rabinowitz P.: On bifurcation from infinity, J. Differential Equations, 14 (3), 462-475 (1973).

23. Rynne B.P.: Bifurcation from zero or infinity in Sturm-Liouville problems which are not linearizable, J. Math.Anal. Appl. 228 (1), 141-156 (1998).

24. Stuart C.A.: Solutions of large norm for non-linear Sturm-Liouville problems, Quart. J. of Math. (Oxford) 24 (2), 129-139 (1973).

25. Toland J.F.: Asymptotic linearity and non-linear eigenvalue problems, Quart. J. Math. (Oxford) 24 (2), 241-250 (1973). 